\title{
Cytoadhesion of Plasmodium falciparum-infected erythrocytes and the infected placenta: a two-way pathway
}

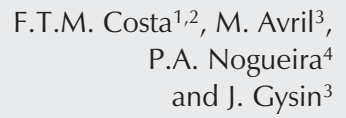

F.T.M. Costa ${ }^{1,2}$, M. Avril ${ }^{3}$,

P.A. Nogueira ${ }^{4}$ and J. Gysin ${ }^{3}$

\author{
${ }^{1}$ Departamento de Microbiologia e Imunologia, \\ ${ }^{2}$ Departamento de Parasitologia, Instituto de Biologia, \\ Universidade Estadual de Campinas, Campinas, SP, Brasil \\ ${ }^{3}$ Unité de Parasitologie Expérimentale, URA Institut Pasteur, \\ Université de la Méditerranée, Marseille, France \\ ${ }^{4}$ Centro de Pesquisa em Medicina Tropical, Porto Velho, RO, Brasil
}

Correspondence

F.T.M. Costa

Departamento de Microbiologia

e Imunologia

Instituto de Biologia, UNICAMP

13083-862 Campinas, SP

Brasil

Fax: +55-19-3788-6276

E-mail: costaftm@unicamp.br

F.T.M. Costa is supported by FAPESP and CNPq-Foundation.

Received January 18, 2006 Accepted August 18, 2006

\section{Abstract}

Malaria is undoubtedly the world's most devastating parasitic disease, affecting 300 to 500 million people every year. Some cases of Plasmodium falciparum infection progress to the deadly forms of the disease responsible for 1 to 3 million deaths annually. P. falciparum-infected erythrocytes adhere to host receptors in the deep microvasculature of several organs. The cytoadhesion of infected erythrocytes to placental syncytiotrophoblast receptors leads to pregnancy-associated malaria (PAM). This specific maternal-fetal syndrome causes maternal anemia, low birth weight and the death of 62,000 to 363,000 infants per year in sub-Saharan Africa, and thus has a poor outcome for both mother and fetus. However, PAM and non-PAM parasites have been shown to differ antigenically and genetically. After multiple pregnancies, women from different geographical areas develop adhesionblocking antibodies that protect against placental parasitemia and clinical symptoms of PAM. The recent description of a new parasite ligand encoded by the $v a r 2^{\mathrm{CSA}}$ gene as the only gene up-regulated in PAM parasites renders the development of an anti-PAM vaccine more feasible. The search for a vaccine to prevent $P$. falciparum sequestration in the placenta by eliciting adhesion-blocking antibodies and a cellular immune response, and the development of new methods for evaluating such antibodies should be key priorities in mother-child health programs in areas of endemic malaria. This review summarizes the main molecular, immunological and physiopathological aspects of PAM, including findings related to new targets in the P. falciparum var gene family. Finally, we focus on a new methodology for mimicking cytoadhesion under blood flow conditions in human placental tissue.
Key words

- Plasmodium falciparum

- Cytoadhesion

- Pregnancy-associated

malaria

- var2 ${ }^{\mathrm{CSA}}$ gene 


\section{Introduction}

Globally, malaria is the most widespread human parasitic disease, affecting 300 to 500 million people per year. Four species of Plasmodium can infect humans: $P$. falciparum, $P$. vivax, $P$. malariae, and $P$. ovale. No complications are observed in most malaria cases, but some $P$. falciparum infections develop into severe forms of the disease, such as cerebral malaria and pregnancy-associated malaria (PAM), which cause more than two million deaths annually. It is estimated that 2.4 billion people, almost half the world's population, are at risk of contracting malaria. In subtropical regions, and subSaharan African countries in particular, this disease limits economic development. The control of this disease has been hampered by the alarming spread of drug-resistant parasites, insecticide-resistant mosquitoes, and the lack of an effective vaccine.

The situation has been aggravated by the deterioration of socioeconomic conditions in rural areas and disordered human migration in countries in which malaria is endemic. These factors have contributed to the re-emergence of malaria. As a result, much of the current research into malaria continues to focus on attempts to develop a vaccine capable of controlling parasite transmission. Some promising results have been obtained, but it seems unlikely that a vaccine conferring significant levels of immune protection, particularly against severe infection, will be developed in the near future.

\section{Severe malaria and Plasmodium falciparum cytoadhesion}

Severe malaria is a multifactorial phenomenon involving the sequestration of $P$. falciparum-infected erythrocytes (IE) in deep vascular beds and the production of inflammatory cytokines, such as TNF- $\alpha$ and IFN- $\gamma$ (1). IE adhere directly to various host endothelial receptors, including CD36, intracel- lular adhesion molecule-1 (ICAM-1), vascular cellular adhesion molecule-1 (VCAM1), E-selectin, P-selectin, hyaluronic acid (HA), and chondroitin sulfate-A (CSA), or to other IE. They may also form rosettes by adhering to non-infected erythrocytes (Figure 1B) (2). It has been suggested that adhesion to host receptors expressed on the surface of endothelial cells enables IE to avoid spleen-mediated filtration and host immune attack, potentially implicating cytoadhesion in parasite survival (3). In addition to direct parasite adhesion to host receptors, platelets can act as a bridge between IE and endothelial cells, providing additional CD36 receptors for cytoadhesion (Figure 1A).

Following infection, $P$. falciparum proteins are exported to the erythrocyte surface, altering host cell conformation and generating electron-dense structures. These structures are known as knobs (Figure 1), and correspond to IE-binding sites adhering to the host endothelium. Knobs are composed of several polypeptides, including $P$. falciparum erythrocyte membrane protein 1 (PfEMP-1; Figure 2A). PfEMP-1 is a variable protein 200 to $350 \mathrm{kDa}$ in size, encoded by the members of the var multigene family, which are present as about 60 distinct copies per haploid parasite genome. These proteins mediate both antigenic variation and cytoadhesion $(2,4)$, and therefore play an important role in parasite virulence. It has been suggested that var gene diversity is largely based on the clustering of these genes at the ends of several chromosomes, creating a "hot-spot" recombination zone facilitating the alignment of homologous sequences located on heterologous chromosomes. Despite this variability, only one antigenic variant is expressed on the surface of the IE at a given time (5). PfEMP-1 contains a transmembrane and an extracellular region, which has been implicated in binding to the cytoadhesion-binding site and as a target for the host immune response (Figure 2A). This extracellular region has a succession of bind- 

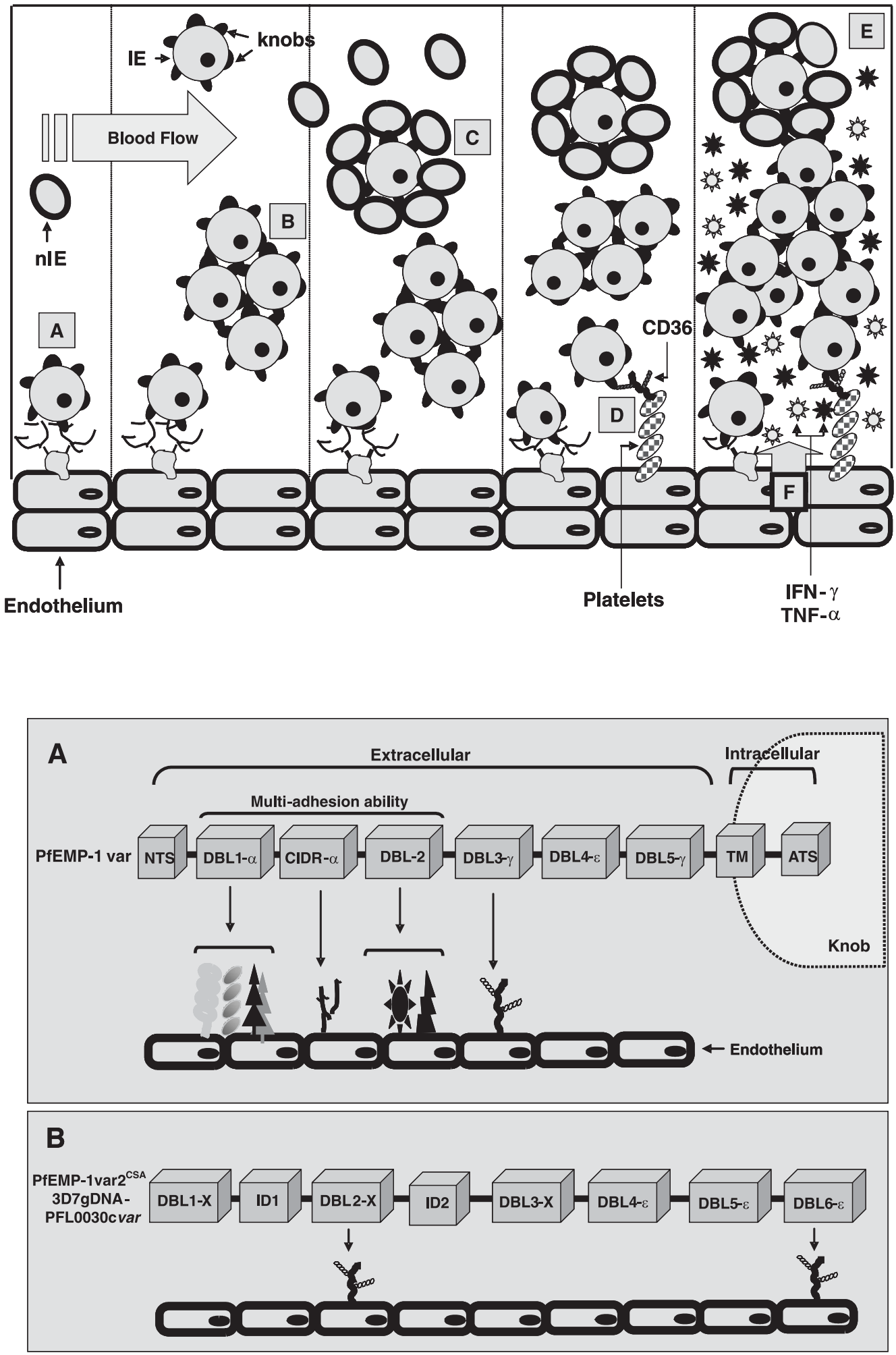

Receptors

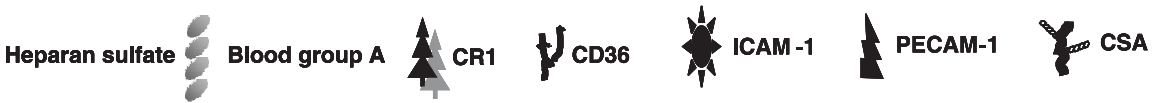

Figure 1. Sequestration mechanisms involved in Plasmodium falciparum infections. $P$. falciparum-infected erythrocytes (IE) adhere directly to different receptors on the host endothelium via knobs (A); to other IE by auto-agglutination (B); to non-infected erythrocytes (nIE), forming rosettes $(C)$; to platelets, which act as a bridge in IE cytoadhesion via the CD36 receptor (D). All these phenomena are thought to contribute to blood flow occlusion (E) and production of the inflammatory cytokines, TNF- $\alpha$ and IFN- $\gamma(\mathrm{F})$; thus leading to the poor clinical outcomes observed in severe malaria.

Figure 2. Schematic structure of Plasmodium falciparum erythrocyte membrane protein-1 (PfEPM1). The intracellular and the immunogenic extracellular regions of a PfEMP-1 are represented. $A$, One PfEMP-1 encoded by a var gene contains several Duffy binding-like (DBL) domains intercalated by cysteine-rich interdomain region domains (CIDR), responsible for mediating parasite cytoadhesion to different receptors directly on the host endothelium, multiadhesion, or adhesion to non-infected erythrocytes, forming rosettes. NTS = $\mathrm{N}$-terminal segment; $\mathrm{TM}=$ transmembrane domain; ATS $=$ acidic teminal segment; CR1 $=$ complement receptor 1 ; ICAM-1 = intracellular adhesion molecule 1; PECAM-1 = platelet endothelial cell adhesion molecule; CSA $=$ chondroitin sulfate $A$. $B$, The PfEMP-1 encoded by the var2 CSA gene 3D7gDNA-PFL0030cvar contains DBL domains capable of CSA-binding and inter-domain regions (ID). 
ing sites arranged in tandem at the $\mathrm{N}$-terminal end of the molecule (Figure 2A). These motifs are known as Duffy binding-like (DBL) domains, as they were first identified in $P$. vivax Duffy binding protein, intercalated by cysteine-rich interdomain region domains (CIDR). Both CIDR and DBL regions can be identified on the basis of their amino-acid sequences $(2,4)$.

Different PfEMP-1 molecules have binding sites for adhesion to different host receptors (Figure 2A), such as CD36, ICAM-1, VCAM-1, E-selectin, P-selectin, CSA, and others dependent on multiple functional binding domains within PfEMP-1. For example, adhesion to CD36, ICAM-1 and CSA is mediated by different PfEMP-1 variants, as var genes are expressed in a mutually exclusive manner, with only one PfEMP-1 expressed on the surface of an IE at a given time (5). Thus, placental parasites can bind CSA, but not the CD36 receptor (6). This dichotomous behavior may result from differences in gene location and transcription orientations between CSA-binding and nonbinding parasites (7-8).

\section{General aspects of PAM}

After years of exposure to the parasite, individuals living in areas of endemic malaria acquire high levels of immunity, limiting parasitemia and attenuating the clinical outcome of malaria. However, pregnant women remain susceptible, especially in their first pregnancy, in which case the risk of contracting malaria is two to ten times higher than that in non-pregnant women living in the same area. Until recently, it was thought that this particular susceptibility of women to malaria during pregnancy was due to pregnancy-related immune suppression and hormonal alterations. However, it has been recently shown that the placenta provides an ideal environment for the development of a subpopulation of malaria parasites that adhere to receptors in the placental syncy- tiotrophoblast. In most cases, the parasites remain on the maternal side of the placenta, but this maternal-fetal syndrome, known as PAM, has adverse effects on both mother and unborn child, causing maternal anemia and low-birth weight (LBW) babies (9). PAM is thought to be responsible for 62,000 to 363,000 infant deaths in sub-Saharan Africa annually (10). Unfortunately, these figures are probably underestimates since peripheral parasitemia is not always observed and the symptoms are not well characterized in some cases.

In PAM, parasite adhesion to CSA, HA and other placental receptors may trigger an inflammatory process involving cytokinesecreting mononuclear cells. The inflammatory component, which may appear after parasite accumulation in the placenta, is associated with the immune-pathological consequences of PAM, such as cytotrophoblast proliferation and cytotrophoblast membrane thickening (10). This inflammatory process, characterized by massive cell deposition, is thought to alter local blood flow, disrupting metabolic pathways and hindering IgG transfer across the placenta and the exchange of nutrients between mother and fetus, resulting in placental lesions and LBW $(10,11)$. However, the clinical outcomes of PAM, such as fetal growth restriction and preterm delivery, the strict association with LBW at term and placental parasitemia, have been observed in primigravidae (9). It is also known that infants born to infected Cameroonian mothers are significantly more susceptible to plasmodial infections, especially in the first two years of life (11). A recent epidemiological analysis in Tanzania, including twice as many infants as the Cameroon study (11), also showed that children born to women with placental malaria presented parasitemia earlier in life than those born to non-infected peers (12). Surprisingly, this study also showed that parity played a role, as the offspring of primigravidae had a significantly lower risk of 
parasitemia than infants born to multigravidae (12). The precise reasons for this remain unclear, but the marked change in cytokine profile and the timing of cytokine production in primigravidae may be involved (12).

Most studies of maternal malaria have been carried out in P. falciparum-infected women. However, pregnant women are susceptible to all four human malaria parasites, including $P$. vivax, the most prevalent parasite in Brazil and elsewhere outside subSaharan Africa. A study in Thailand revealed that primigravidae had a significantly higher risk of $P$. vivax infection than multigravidae. Moreover, $P$. vivax infection has also been shown to be significantly associated with maternal anemia and risk of LBW, although these outcomes were more marked in multigravidae (13). The deposition of malaria pigment in the placenta has been observed in $P$. vivax-infected women (14), and variant antigens encoded by a specific $P$. vivax multigene family have been identified (15). However, hard data on $P$. vivax cytoadhesion to the placental syncytiotrophoblast or endothelial cells remain scarce.

In Brazil, where malaria transmission is unstable, $P$. falciparum and $P$. vivax infections account for 15.1 and $84.4 \%$ of cases among non-pregnant women (16). However, the corresponding proportions for pregnant women are $29.7 \%$ for falciparum and $67.7 \%$ for vivax malaria (16). This corresponds to a significant, 2.5 times increase in the frequency of $P$. falciparum infection for the 195 cases of malaria in pregnant women analyzed (16). The precise reason for this shift in prevalence is unclear and further studies with a larger number of patients are required.

\section{Ligands and receptors involved in PAM}

The DBL- $\gamma 3$ domain of PfEMP-1, encoded by the $\operatorname{var} 1^{\mathrm{CSA}}$ gene, was initially thought to be the ligand responsible for parasite cytoadhesion in PAM (17). However, several recent studies have suggested that the protein encoded by the var2 ${ }^{\mathrm{CSA}}$ gene may be the principal ligand involved in placental cytoadhesion. Unlike $\operatorname{var}{ }^{\mathrm{CSA}}$, $\operatorname{var} 2^{\mathrm{CSA}}$ is up-regulated in placental parasites after selection for adhesion to the CSA receptor in vitro (18-20); indeed it is the only gene shown to be transcriptionally up-regulated following such selection. var $2^{\mathrm{CSA}}$ knockout parasites are unable to recover their initial CSA binding, even after repeated exposure to CSA $(21,22)$. The PfEMP-1 encoded by the $\operatorname{var} 2^{\mathrm{CSA}}$ gene has been shown to have a structure different from that of other var genes, in that it lacks the CIDR, DBL- $\gamma$ and $N$-terminal DBL- $\alpha$ domains (Figure 2B). The PfEMP-1 encoded by the 3D7 var2 ${ }^{\text {CSA }}$ gene (3D7gDNA-PFL0030cvar) has six DBL motifs: DBL4- $\varepsilon$, DBL5- $\varepsilon$ and DBL6- $\varepsilon$, and a further three motifs that do not fit into the current classification: DBL1-X, DBL2$X$ and DBL3-X. The DBL2-X and DBL6- $\varepsilon$ domains are able to bind CSA (Figure 2B) (18).

A recent mass spectrometry-based proteomics study identified novel parasite antigens, which might be expressed on the IE surface, exclusively in CSA-binding or placental parasites, but did not evaluate the binding of these antigens to host receptors (23).

There is evidence that CSA is not the only placental receptor and that a subpopulation of parasites collected from infected placentas may also bind to HA and to nonimmune IgG via their F(ab') moieties. However, recent cytoadhesion assays using placental parasites collected from 60 pregnant Tanzanian women with malaria showed that almost all placental parasites capable of binding to at least one host receptor were also able to adhere to immobilized CSA, and that only three of 46 of these parasites adhered to immobilized HA. In binding inhibition assays using soluble CSA as a competitor, 
adhesion to placental sections was significantly abolished in all placental parasites tested. In contrast, soluble HA, non-immune IgG and protein A failed to inhibit parasite binding to placental cryosections. These findings strongly suggest that CSA is the major placental receptor, and support the development of vaccines targeting parasite ligands to CSA (24).

The CSA or chondroitin 4-sulfate (C4S) receptor is a glycosaminoglycan present in the extracellular matrix, and was first identified as a receptor for parasite binding to Saimiri monkey endothelial brain cells and to Chinese hamster ovary cells $(25,26)$. Gysin et al. (27) showed that thrombomodulin with a CSA chain was the dominant proteoglycan involved in the sequestration of CSA-binding parasites in the placenta and that a CSA chain at least $9 \mathrm{kDa}$ in size was required to sustain the adhesion of CSA-binding parasites (28). Achur et al. (29) subsequently purified and identified several types of chondroitin sulfate proteoglycan (CSPG) from the human placenta, showing that these natural CSPGs present in the intervillous space contained unusually low levels of sulfate and served as receptors for PAM parasite adhesion. The same group went on to define the structures required for parasite cytoadhesion as $\mathrm{C} 4 \mathrm{~S}$ dodecasaccharides, and showed that these placental CSPGs have a unique distribution of sulfate groups throughout the second and third semesters of pregnancy (30-32). It was recently shown that the ability of antiadhesive molecules to inhibit $\mathrm{C} 4 \mathrm{~S}$-specific binding also depends on the sulfation partner of these CSPGs (33).

\section{Does the antibody-mediated immune response play a role in PAM?}

Despite pregnancy-related immunosuppression, pregnant women with malaria develop antibodies that inhibit the binding of IE to CSA, and these antibodies are associ- ated with protection against placental infection. Primigravidae have a much higher susceptibility to maternal malaria than multigravidae, because the antibodies acquired after multiple pregnancies are associated with a reduction in the number of IE in the placenta (34). In addition, higher levels of these CSA adhesion-blocking antibodies are correlated with less pronounced maternal anemia and with higher birth weight for babies born at term $(35,36)$.

For var2 ${ }^{\mathrm{CSA}}$ parasites, a recent study showed that rabbit antibodies raised against two VAR2CSA recombinant proteins, corresponding to the DBL1-X and DBL5- $\varepsilon$ domains, recognize only the surface proteins of PAM parasites (20). Plasma samples from Ghanaian individuals recognized these recombinant proteins in a sex- and paritydependent manner in ELISA tests; this was particularly true for the recombinant protein based on the DBL5- $\varepsilon$ domain (20). High plasma levels of anti-VAR2CSA antibodies in women were also found to be correlated with a lower risk of LBW (20). Finally, monoclonal antibodies (mAbs) that recognize VAR2CSA DBL domains inhibit, to various extents, the adhesion of a placental isolate to placental cryosections under flow conditions. Moreover, sera from mice immunized with native VAR2CSA domain complexes with specific mAbs strongly inhibit PAM parasite cytoadhesion to CSA on the surface of endothelial cells (37).

These observations suggest that one probable mechanism controlling placental parasitemia and attenuating clinical outcome may be based on adhesion-blocking antibodies against CSA-binding domains. However, as primigravidae and multigravidae present significant levels of adhesion-blocking antibodies at term, the timing of acquisition of these antibodies may be important in immune protection. O'Neil-Dunne et al. (38) studied 198 pregnant Cameroonian women and showed that multigravidae began mounting an antibody-based immune response af- 
ter just 12 weeks of gestation, whereas primigravidae took eight weeks longer to develop such adhesion-blocking antibodies. However, as cytophilic antibodies have been collected from infected pregnant women (39), it should be borne in mind that other antibodydependent mechanisms, such as phagocytosis and complement activation, may also help to protect against PAM, in addition to blocking adhesion. Consistent with the existence of additional antibody mechanisms, Megnekou et al. (40) showed that IgG1 and IgG3 were the most prevalent subclasses of PAM antibodies in Cameroonian women, and that larger amounts of these antibodies were found in pregnant multiparous women.

\section{Is the cell-mediated immune response involved in PAM pathogenesis or protection?}

The precise mechanism by which PAM parasites evade the immune system and the possible involvement of a cell-mediated immune response in protection remains unresolved. However, it has been shown that massive sequestration of the parasite in the placenta leads to a switch in the cell-mediated immune response, typically from $\mathrm{T}_{\mathrm{H}} 2$ to $T_{H} 1$, resulting in the clinical manifestation of PAM, characterized by an increase in the level of pro-inflammatory cytokine production (10). Several studies have shown that high levels of IFN- $\gamma$ and TNF- $\alpha$, mainly secreted by placental macrophages, are associated with poor clinical outcome in patients with PAM and with the concentration of hemozoin in the placenta (41). Placental infection increases the levels of $\alpha$ - and $\beta$ chemokines, which, in turn, increase immune cell recruitment to the placenta $(10,42)$.

In contrast, IFN- $\gamma$ levels have been reported to be higher following in vitro stimulation of blood mononuclear cells from multigravidae than following the stimulation of such cells from women in their first or second pregnancy. The cells collected from women in their second pregnancy secreted high levels of IL-4 and IL-10 (43). Multigravidae have been shown to present higher levels of lymphocyte proliferation and natural killer cell cytotoxic activity in response to CSA-binding parasites than primigravidae women (10). These observations suggest that IFN- $\gamma$ is involved in immunity to PAM. Further evidence for the protective role of IFN- $\gamma$ is provided by the higher susceptibility to PAM of women with both malaria and HIV infection (10). Indeed, it has been recently shown that neonates born to mothers with active placental infection have lower levels of PAM-parasite antigen-specific IFN$\gamma$ T cells and higher levels of IL-10 CD4 T cells than do pregnant infected women treated for malaria (44). However, TGF- $\beta$, an antiinflammatory cytokine, is produced in larger amounts in multigravidae than in primigravidae, suggesting a possible role in controlling the manifestation of PAM clinical symptoms.

A recent study in monkeys showed that infection with $P$. coatneyi did not result in higher levels of CD4 and CD8 T lymphocytes than observed in infected non-pregnant monkeys. Indeed, the pregnant infected monkeys had lower levels of monocytes and macrophages in peripheral blood than did the non-pregnant infected monkeys (45). Conversely, high levels of mononuclear cell accumulation have been associated with poor PAM outcomes (46). It should be noted that, in these studies, cells were counted in peripheral blood, so we cannot exclude the possibility that this modulation may alter the levels of these cells in placental compartments. In PAM, T cells collected from peripheral blood proliferate more efficiently than those collected from the intervillous blood, whereas intervillous and peripheral monocytes are equally able to present antigens (47).

These observations suggest a dual effect on cytokine production in PAM and that a fine balance in the timing and levels of pro- 
and anti-inflammatory cytokines dictates whether an individual will manage to control PAM or whether the clinical symptoms associated with the disease will develop. This dual effect may depend on regulation of the macrophage migration inhibitory factor, a specific cytokine that counter-regulates the immunosuppressive effects of pregnancy. Placental infection was recently shown to increase macrophage migration inhibitory factor production in the presence of cytotrophoblast-adherent IE (48).

\section{Is an anti-PAM vaccine feasible?}

The first evidence that it might be possible to develop an anti-PAM vaccine was provided by the study of Fried and Duffy $(9,35)$ and Staalsoe et al. $(34,36)$ showing that multiparous women were less susceptible to PAM than women in their first pregnancy, that infected women developed high levels of adhesion-blocking antibodies against PAM parasites after several pregnancies $(9,34)$ and that these antibodies were associated with attenuation of the clinical outcome of PAM. Antibodies against var2 ${ }^{\mathrm{CSA}}$ parasites have also been shown to crossreact with genetically different $P$. falciparum strains (49). Cross-reactivity between the DBL- $\gamma 3^{\mathrm{CSA}}$ domain and var $2^{\mathrm{CSA}}$-encoded antigens has been observed (50). Furthermore, mAbs raised against var $2^{\mathrm{CSA}}$ parasite surface antigens have also proved to be panreactive with CSA-binding parasites from different geographical origins (37). Moreover, molecular analysis of the var $1^{\mathrm{CSA}}$ DBL$\gamma 3$ minimal binding domain revealed 37\% sequence identity to the var $2^{\mathrm{CSA}}$ DBL3-X domain (51). These somewhat surprising panreactivity results are probably related to conformational similarities.

In light of the antibody-mediated immune response in PAM, an efficient vaccine would probably elicit large amounts of adhesion-blocking antibodies, mainly against conserved binding motifs. However, as the cell-mediated immune response also seems to be involved in immune protection against PAM, immunization regimes and adjuvants should aim to induce high levels of IFN- $\gamma$ secreting $\mathrm{T}$ cells. Immunization regimens based on naked DNA for priming and recombinant viral vectors or proteins for boosting have been shown to elicit high levels of CD4- and CD8-producing T cells able to induce immune protection against several viral and protozoan diseases (52). Finally, anti-PAM vaccines may contain other parasite antigens since sera collected from Cameroonian women showed a significant correlation between low or null levels of antibodies against the carboxyl-terminal $19-\mathrm{kDa}$ segment of the $P$. falciparum merozoite surface protein- 1 and the risk of PAM (53).

\section{Is it possible to model PAM?}

Reliable in vitro adhesion models are required for the evaluation of potential vaccine candidates and the antibody-mediated immune response directed against them. Most of the existing in vitro models of IE sequestration were developed for studying IE adhesion in parasites thought to be involved in cerebral malaria. Many knob $^{+}$laboratoryadapted $P$. falciparum strains adhere in vitro to various cell types, including human umbilical vein endothelial cells, C32 amelanotic melanoma cells, human dermal microvascular endothelial cells, human brain capillary endothelial cells, human monocytes and platelets, and transfected COS cells (54). However, in 1995, Gay et al. (55) described the use of Saimiri microvascular brain endothelial cell clones differing in terms of the expression of several combined surface molecules such as CD36, ICAM-1, E-selectin, and CSA, permitting for the first time the selection by cytoadhesion of distinct monomorphic adhesion phenotypes.

Each model attempts to simulate the interaction between IE and the cerebral endothelial cells, but none can be considered 
ideal. The Saimiri cell model has some advantages in that it allows in vitro cytoadhesion studies, the results of which can be confirmed or rejected using the homologous Saimiri/P. falciparum monkey model (56). This model is also considered to be more relevant than non-primate cell models due to the phylogenetic proximity to humans. The use of organ-specific endothelial cells appears to be particularly useful for structurefunction studies in which the native conformation of a receptor is critical. A placental BeWo-derived cell line has been successfully used to select monomorphic CSA-binding parasites (57). Since placental CSPGs have an unusual sulfation pattern, BeWo cells provide an alternative to cells of nonplacental origin in CSA-binding studies.

In all the parasite-binding assays described above, cytoadhesion was investigated under static conditions, in which suspensions of IE were allowed to settle on a confluent monolayer of cultured cells. This method is technically simple, making it possible to carry out a large number of assays simultaneously. However, static assays do not model the dynamic blood flow conditions encountered by IE in vivo.

In 1995, Cooke and Coppel (54) developed an in vitro assay for visualizing and quantifying the adhesion of IE to endothelial cells or to immobilized adhesion receptors under flow conditions. The flow assembly used consisted of a parallel-plate flow chamber or a glass microcapillary tube (microslide) on which a monolayer of endothelial cells, such as human umbilical vein endothelial cells, C32 melanoma cells, or Saimiri microvascular brain endothelial cell clones (58), can be cultured, or a plastic slide coated with purified proteins, such as CD36, ICAM-1 or thrombospondin, by adsorption. Adhesion under dynamic flow conditions can be quantified by counting adherent IE directly under the microscope.

Nevertheless, with the exception of this flow adhesion model using endothelial cells and immobilized proteins, very few models have been developed for studying placental malaria. One example is the Saimiri (squirrel monkey) microvascular endothelial cell line Sc17, which expresses a thrombomodulin bearing only a CSA chain and a CD44csa isoform. The presence of the chondroitin sulfate of thrombomodulin, or a CD44 isoform, on endothelial cells mimics, to some extent, the presence of CSA on the surface of the syncytiotrophoblast, thereby providing a clear advantage over previous cell models or commercial CSA preparations from various non-placental sources (59). Conversely, one major disadvantage of the use of cell lines as models for CSA binding is the presence of unidentified or unknown adhesion receptors in addition to CSA on the surface of endothelial cells. Another disadvantage of these assays is that CSA preparations from various sources, used by different laboratories, can generate conflicting results. Thus, the conformational modification of CSA by adding dipalmitoyl-diphosphatidylethanolamine may bias results, especially when this system is used to select CSA-binding IE by panning. The addition of charged groups seems to be problematic for the specific selection of CSA-binding IE from laboratory strains and field isolates (28). Furthermore, cytoadhesion inhibition assays with Saimiri brain endothelial or Chinese hamster ovary cells cannot distinguish between subpopulations of CSA-binding parasites in field samples.

However, most of these problems can be solved by using normal and at-term human placental cryosections (60), as IE bind almost exclusively to syncytiotrophoblast and in the intervillous space containing proteoglycans with low levels of sulfation (61). The use of human placental tissue makes it much easier to count IE under flow than under static conditions, in which parasites also bind to CSA and to other receptors within the villi. A comparison of inhibition assays carried out under flow and static con- 
ditions revealed significant differences in the presence of soluble CSA, or inhibitor mAbs in serum samples from primi- and multigravidae (61). Static parasite adhesion assays are subject to considerable inter-experimental variation, due primarily to differences in washing procedures.

The use of placental cryosections made it possible, for the first time, to measure the shear-stress resistance of CSA-binding IE. Distinct subpopulations within the CSAbinding phenotype were identified by increasing the flow rate gradually from 0.2 to 3.2 Pascal (Pa). For example, at 0.6 Pa, which exceeds the normal shear stress in the placenta $(0.05 \mathrm{~Pa}), 70 \%$ of IE remained adherent for the laboratory strain FCR3 ${ }^{\mathrm{CSA}}$, and $25 \%$ of IE resisted a shear stress $>3.2 \mathrm{~Pa}$ (60).

These results strongly suggest that the initial FCR3 ${ }^{\text {CSA }}$ strain was composed of a mixture of strong $(\geq 3.2 \mathrm{~Pa})$ and weak $(\leq 0.8$ $\mathrm{Pa}$ CSA-binding parasites, confirming the existence of distinct adhesion subpopulations among the CSA phenotypes of various strains (58; Nogueira PA, Costa FT and Gysin $\mathrm{J}$, unpublished data), and supporting the hypothesis that only some subpopulations of CSA-binding IE have the potential for sequestration in the microvasculature (58). This hypothesis is based on the notion that IE in the placenta are not normally exposed to shear stresses exceeding $0.05 \mathrm{~Pa}$, whereas shear stresses in the postcapillary venules vary from 0.1 to $1 \mathrm{~Pa}(58)$.

\section{Conclusions}

There is now considerable evidence that PAM is a particularly severe form of malaria, and that primigravidae and their offspring have a higher risk of developing PAM than do multigravidae and their children. These observations, and others, provide an impetus for the development of an anti-PAM vaccine. However, several issues concerning the expression of antigens by PAM parasites and the precise immunological mechanisms involved in protection remain unresolved in the context of PAM. First, does the unique set of hormones and cytokines induced during gestation play a role in antigen or placental host receptor expression? Second, why is CSA the major PAM receptor, given that this glycosaminoglycan is found in various organs other than the placenta? Third, how many antigenically different PAM parasites exist, and what are their relative prevalences in infected pregnant women? Fourth, what is the evolutionary importance of this pan-reactivity and the presence of multiple CSA-binding domains? Can these domains be ordered into a hierarchy? Finally, as some of the poor placental outcomes observed in falciparum malaria are also observed in vivax malaria, is IE cytoadhesion in the placenta an exclusive feature of $P$. falciparum parasites? Or, like the inhabitants of "Plato's Cave", are we merely watching the "theater of shadows" of real PAM parasite interactions and mechanisms reflected on the cave wall?

\section{Acknowledgments}

We would like to thank Dr. Artur Scherf (Institut Pasteur, Paris, France) and Dr. Daniela D. Carvalho (Universidade Estadual de Campinas, Campinas, SP, Brazil) for critically reading the manuscript and Rafael Chaves (Universidade Estadual de Campinas, Campinas, SP, Brazil) for editorial assistance. We would like to apologize to those authors whose published articles have not been cited in this review due to space constraints and the huge body of literature available. 


\section{References}

1. Schofield L, Grau GE. Immunological processes in malaria pathogenesis. Nat Rev Immunol 2005; 5: 722-735.

2. Wahlgren M, Treutiger CJ, Gysin J. Cytoadherence and rosetting in pathogenesis of severe malaria. In: Wahlgren M, Perlmann P (Editors), Malaria molecular and clinical aspects. The Netherlands: Harwood Academic Publishers; 1999. p 289-328.

3. David PH, Hommel M, Miller LH, Udeinya IJ, Oligino LD. Parasite sequestration in Plasmodium falciparum malaria: spleen and antibody modulation of cytoadherence of infected erythrocytes. Proc Natl Acad Sci U S A 1983; 80: 5075-5079.

4. Winter G, Chen $Q$, Wahlgren M. Meeting report: the molecular background of severe and complicated malaria. Mol Biochem Parasitol 2004; 134: 37-41.

5. Freitas-Junior LH, Bottius E, Pirrit LA, Deitsch KW, Scheidig C, Guinet $F$, et al. Frequent ectopic recombination of virulence factor genes in telomeric chromosome clusters of $P$. falciparum. Nature 2000; 407: 1018-1022.

6. Gamain B, Gratepanche S, Miller LH, Baruch DI. Molecular basis for the dichotomy in Plasmodium falciparum adhesion to CD36 and chondroitin sulfate A. Proc Natl Acad Sci U S A 2002; 99: 1002010024.

7. Robinson BA, Welch TL, Smith JD. Widespread functional specialization of Plasmodium falciparum erythrocyte membrane protein 1 family members to bind CD36 analysed across a parasite genome. Mol Microbiol 2003; 47: 1265-1278.

8. Kraemer SM, Smith JD. Evidence for the importance of genetic structuring to the structural and functional specialization of the Plasmodium falciparum var gene family. Mol Microbiol 2003; 50: 15271538.

9. Duffy PE, Fried M. Malaria in the pregnant woman. Curr Top Microbiol Immunol 2005; 295: 169-200.

10. Beeson JG, Duffy PE. The immunology and pathogenesis of malaria during pregnancy. Curr Top Microbiol Immunol 2005; 297: 187-227.

11. Le Hesran JY, Cot M, Personne P, Fievet N, Dubois B, Beyeme M, et al. Maternal placental infection with Plasmodium falciparum and malaria morbidity during the first 2 years of life. Am $J$ Epidemiol 1997; 146: 826-831.

12. Mutabingwa TK, Bolla MC, Li JL, Domingo GJ, Li X, Fried M, et al. Maternal malaria and gravidity interact to modify infant susceptibility to malaria. PLoS Med 2005; 2: e407.

13. Nosten F, McGready R, Simpson JA, Thwai KL, Balkan S, Cho T, et al. Effects of Plasmodium vivax malaria in pregnancy. Lancet 1999; 354: 546-549.

14. McGready R, Davison BB, Stepniewska K, Cho T, Shee H, Brockman A, et al. The effects of Plasmodium falciparum and $P$. vivax infections on placental histopathology in an area of low malaria transmission. Am J Trop Med Hyg 2004; 70: 398-407.

15. Del Portillo HA, Fernandez-Becerra C, Bowman S, Oliver K, Preuss $M$, Sanchez CP, et al. A superfamily of variant genes encoded in the subtelomeric region of Plasmodium vivax. Nature 2001; 410: 839842.

16. Martinez-Espinosa FE, David PH, Daniel-Ribeiro CT, Alecrim WD. Malaria during pregnancy in a reference centre from the Brazilian Amazon: unexpected increase in the frequency of Plasmodium falciparum infections. Mem Inst Oswaldo Cruz 2004; 99: 19-21.

17. Buffet PA, Gamain B, Scheidig C, Baruch D, Smith JD, HernandezRivas $\mathrm{R}$, et al. Plasmodium falciparum domain mediating adhesion to chondroitin sulfate $A$ : a receptor for human placental infection. Proc Natl Acad Sci U S A 1999; 96: 12743-12748.
18. Gamain B, Trimnell AR, Scheidig C, Scherf A, Miller LH, Smith JD. Identification of multiple chondroitin sulfate A (CSA)-binding domains in the var2CSA gene transcribed in CSA-binding parasites. $J$ Infect Dis 2005; 191: 1010-1013.

19. Salanti A, Staalsoe T, Lavstsen T, Jensen AT, Sowa MP, Arnot DE, et al. Selective upregulation of a single distinctly structured var gene in chondroitin sulphate A-adhering Plasmodium falciparum involved in pregnancy-associated malaria. Mol Microbiol 2003; 49: 179-191.

20. Salanti A, Dahlback M, Turner L, Nielsen MA, Barfod L, Magistrado $\mathrm{P}$, et al. Evidence for the involvement of VAR2CSA in pregnancyassociated malaria. J Exp Med 2004; 200: 1197-1203.

21. Andrews KT, Pirrit LA, Przyborski JM, Sanchez CP, Sterkers $Y$, Ricken $\mathrm{S}$, et al. Recovery of adhesion to chondroitin-4-sulphate in Plasmodium falciparum varCSA disruption mutants by antigenically similar PfEMP1 variants. Mol Microbiol 2003; 49: 655-669.

22. Viebig NK, Gamain B, Scheidig C, Lepolard C, Przyborski J, Lanzer $\mathrm{M}$, et al. A single member of the Plasmodium falciparum var multigene family determines cytoadhesion to the placental receptor chondroitin sulphate A. EMBO Rep 2005; 6: 775-781.

23. Fried M, Wendler JP, Mutabingwa TK, Duffy PE. Mass spectrometric analysis of Plasmodium falciparum erythrocyte membrane protein- 1 variants expressed by placental malaria parasites. Proteomics 2004; 4: 1086-1093.

24. Fried M, Domingo GJ, Gowda CD, Mutabingwa TK, Duffy PE. Plasmodium falciparum: chondroitin sulfate $A$ is the major receptor for adhesion of parasitized erythrocytes in the placenta. Exp Parasitol 2006; 113: 36-42.

25. Rogerson SJ, Chaiyaroj SC, Ng K, Reeder JC, Brown GV. Chondroitin sulfate $A$ is a cell surface receptor for Plasmodium falciparum-infected erythrocytes. J Exp Med 1995; 182: 15-20.

26. Pouvelle B, Meyer P, Robert C, Bardel L, Gysin J. Chondroitin-4sulfate impairs in vitro and in vivo cytoadherence of Plasmodium falciparum infected erythrocytes. Mol Med 1997; 3: 508-518.

27. Gysin J, Pouvelle B, Le Tonqueze M, Edelman L, Boffa MC. Chondroitin sulfate of thrombomodulin is an adhesion receptor for Plasmodium falciparum-infected erythrocytes. Mol Biochem Parasitol 1997; 88: 267-271.

28. Pouvelle B, Fusai T, Lepolard C, Gysin J. Biological and biochemical characteristics of cytoadhesion of Plasmodium falciparum-infected erythrocytes to chondroitin-4-sulfate. Infect Immun 1998; 66: 4950-4956.

29. Achur RN, Valiyaveettil M, Alkhalil A, Ockenhouse CF, Gowda DC. Characterization of proteoglycans of human placenta and identification of unique chondroitin sulfate proteoglycans of the intervillous spaces that mediate the adherence of Plasmodium falciparum-infected erythrocytes to the placenta. J Biol Chem 2000; 275: 4034440356.

30. Alkhalil A, Achur RN, Valiyaveettil M, Ockenhouse CF, Gowda DC. Structural requirements for the adherence of Plasmodium falciparum-infected erythrocytes to chondroitin sulfate proteoglycans of human placenta. J Biol Chem 2000; 275: 40357-40364.

31. Achur RN, Valiyaveettil M, Gowda DC. The low sulfated chondroitin sulfate proteoglycans of human placenta have sulfate group-clustered domains that can efficiently bind Plasmodium falciparuminfected erythrocytes. J Biol Chem 2003; 278: 11705-11713.

32. Agbor-Enoh ST, Achur RN, Valiyaveettil M, Leke R, Taylor DW, Gowda DC. Chondroitin sulfate proteoglycan expression and binding of Plasmodium falciparum-infected erythrocytes in the human placenta during pregnancy. Infect Immun 2003; 71: 2455-2461. 
33. Andrews KT, Klatt N, Adams Y, Mischnick P, Schwartz-Albiez R. Inhibition of chondroitin-4-sulfate-specific adhesion of Plasmodium falciparum-infected erythrocytes by sulfated polysaccharides. Infect Immun 2005; 73: 4288-4294.

34. Staalsoe T, Megnekou R, Fievet N, Ricke CH, Zornig HD, Leke R, et al. Acquisition and decay of antibodies to pregnancy-associated variant antigens on the surface of Plasmodium falciparum-infected erythrocytes that protect against placental parasitemia. $J$ Infect Dis 2001; 184: 618-626.

35. Duffy PE, Fried M. Antibodies that inhibit Plasmodium falciparum adhesion to chondroitin sulfate $A$ are associated with increased birth weight and the gestational age of newborns. Infect Immun 2003; 71 : 6620-6623.

36. Staalsoe T, Shulman CE, Bulmer JN, Kawuondo K, Marsh K, Hviid L. Variant surface antigen-specific IgG and protection against clinical consequences of pregnancy-associated Plasmodium falciparum malaria. Lancet 2004; 363: 283-289.

37. Avril M, Gamain B, Lépolard C, Viaud N, Scherf A, Gysin J. Mapping of var2CSA-DBL domains recognized by mouse monoclonal antibodies that inhibit adhesion to chondroitin sulfate A. Microbes Infect 2006 (in press).

38. O'Neil-Dunne I, Achur RN, Agbor-Enoh ST, Valiyaveettil M, Naik RS, Ockenhouse CF, et al. Gravidity-dependent production of antibodies that inhibit binding of Plasmodium falciparum-infected erythrocytes to placental chondroitin sulfate proteoglycan during pregnancy. Infect Immun 2001; 69: 7487-7492.

39. Gysin J, Pouvelle B, Fievet N, Scherf A, Lepolard C. Ex vivo desequestration of Plasmodium falciparum-infected erythrocytes from human placenta by chondroitin sulfate A. Infect Immun 1999; 67: 6596-6602.

40. Megnekou R, Staalsoe T, Taylor DW, Leke R, Hviid L. Effects of pregnancy and intensity of Plasmodium falciparum transmission on immunoglobulin $G$ subclass responses to variant surface antigens. Infect Immun 2005; 73: 4112-4118.

41. Fried M, Muga RO, Misore AO, Duffy PE. Malaria elicits type 1 cytokines in the human placenta: IFN-gamma and TNF-alpha associated with pregnancy outcomes. J Immunol 1998; 160: 2523-2530.

42. Suguitan AL Jr, Leke RG, Fouda G, Zhou A, Thuita L, Metenou S, et al. Changes in the levels of chemokines and cytokines in the placentas of women with Plasmodium falciparum malaria. J Infect Dis 2003; 188: 1074-1082.

43. Moore JM, Nahlen BL, Misore A, Lal AA, Udhayakumar V. Immunity to placental malaria. I. Elevated production of interferon-gamma by placental blood mononuclear cells is associated with protection in an area with high transmission of malaria. J Infect Dis 1999; 179: 1218-1225.

44. Brustoski K, Moller U, Kramer M, Petelski A, Brenner S, Palmer DR, et al. IFN-gamma and IL-10 mediate parasite-specific immune responses of cord blood cells induced by pregnancy-associated Plasmodium falciparum malaria. J Immunol 2005; 174: 1738-1745.

45. Davison BB, Kaack MB, Rogers LB, Rasmussen KK, Rasmussen T, Henson EW, et al. Alterations in the profile of blood cell types during malaria in previously unexposed primigravid monkeys. J Infect Dis 2005; 191: 1940-1952.

46. Ordi J, Ismail MR, Ventura PJ, Kahigwa E, Hirt R, Cardesa A, et al. Massive chronic intervillositis of the placenta associated with malaria infection. Am J Surg Pathol 1998; 22: 1006-1011.

47. Diouf I, Fievet N, Doucoure S, Ngom M, Gaye A, Dumont A, et al.
Monocyte activation and T cell inhibition in Plasmodium falciparuminfected placenta. J Infect Dis 2004; 189: 2235-2242.

48. Chaisavaneeyakorn S, Lucchi N, Abramowsky C, Othoro C, Chaiyaroj SC, Shi YP, et al. Immunohistological characterization of macrophage migration inhibitory factor expression in Plasmodium falciparum-infected placentas. Infect Immun 2005; 73: 3287-3293.

49. Elliott SR, Duffy MF, Byrne TJ, Beeson JG, Mann EJ, Wilson DW, et al. Cross-reactive surface epitopes on chondroitin sulfate A-adherent Plasmodium falciparum-infected erythrocytes are associated with transcription of var2csa. Infect Immun 2005; 73: 2848-2856.

50. Bir N., Yazdani S.S., Avril M., Layez C., Gysin J., Chitnis C. Immunogenicity of Duffy binding-like domains that bind chondroitin sulfate and protection against pregnancy-associated malaria. Infct Immn. 2006; 74; 5955-5963.

51. Gamain B, Smith JD, Avril M, Baruch DI, Scherf A, Gysin J, et al. Identification of a 67-amino-acid region of the Plasmodium falciparum variant surface antigen that binds chondroitin sulphate $A$ and elicits antibodies reactive with the surface of placental isolates. $\mathrm{Mol}$ Microbiol 2004; 53: 445-455.

52. Todryk SM, Walther M. Building better T-cell-inducing malaria vaccines. Immunology 2005; 115: 163-169.

53. Taylor DW, Zhou A, Marsillio LE, Thuita LW, Leke EB, Branch O, et al. Antibodies that inhibit binding of Plasmodium falciparum-infected erythrocytes to chondroitin sulfate $A$ and to the $C$ terminus of merozoite surface protein 1 correlate with reduced placental malaria in Cameroonian women. Infect Immun 2004; 72: 1603-1607.

54. Cooke BM, Coppel RL. Cytoadhesion and falciparum malaria: going with the flow. Parasitol Today 1995; 11: 282-287.

55. Gay F, Robert C, Pouvelle B, Peyrol S, Scherf A, Gysin J. Isolation and characterization of brain microvascular endothelial cells from Saimiri monkeys. An in vitro model for sequestration of Plasmodium falciparum-infected erythrocytes. J Immunol Methods 1995; 184 : 15-28.

56. Gysin J, Aikawa M, Tourneur N, Tegoshi T. Experimental Plasmodium falciparum cerebral malaria in the squirrel monkey Saimiri sciureus. Exp Parasitol 1992; 75: 390-398.

57. Viebig NK, Nunes MC, Scherf A, Gamain B. The human placental derived BeWo cell line: a useful model for selecting Plasmodium falciparum CSA-binding parasites. Exp Parasitol 2006; 112: 121 125.

58. Pouvelle B, Traore B, Nogueira PA, Pradines B, Lepolard C, Gysin J. Modeling of Plasmodium falciparum-infected erythrocyte cytoadhesion in microvascular conditions: chondroitin-4-sulfate binding, $A$ competitive phenotype. J Infect Dis 2003; 187: 292-302.

59. Fusai T, Parzy D, Spillmann D, Eustacchio F, Pouvelle B, Lepolard $\mathrm{C}$, et al. Characterisation of the chondroitin sulphate of Saimiri brain microvascular endothelial cells involved in Plasmodium falciparum cytoadhesion. Mol Biochem Parasitol 2000; 108: 25-37.

60. Avril M, Traore B, Costa FT, Lepolard C, Gysin J. Placenta cryosections for study of the adhesion of Plasmodium falciparuminfected erythrocytes to chondroitin sulfate $A$ in flow conditions. Microbes Infect 2004; 6: 249-255.

61. Muthusamy A, Achur RN, Bhavanandan VP, Fouda GG, Taylor DW, Gowda DC. Plasmodium falciparum-infected erythrocytes adhere both in the intervillous space and on the villous surface of human placenta by binding to the low-sulfated chondroitin sulfate proteoglycan receptor. Am J Pathol 2004; 164: 2013-2025. 